\title{
УДК 721.925
}

\section{FEATURES OF THE IMPLEMENTATION OF BIM TECHNOLOGIES IN THE DESIGN DEVELOPMENT PROCESS OF ARCHITECTURAL \\ COMPANIES}

\section{ОСОБЕННОСТИ ВНЕДРЕНИЕ ВІМ ТЕХНОЛОГИЙ В ПРОЦЕСС ПРОЕКТНЫХ РАЗРАБОТОК АРХИТЕКТУРНЫХ КОМПАНИЙ}

Shadibekov T.K. / Шадыбеков T.X. student /cтудент

Kazakh Leading Academy of Architecture and Civil Engineering, Almaty, Ryskulbekova, 28

Казахская головная архитектурно-строительная академия, Алматы, Рыскулбекова, 28

\begin{abstract}
Аннотация. B работе рассматриваются сложности прочессы перехода на параметрическое проектирование архитектурных компаний, связанные с особенностями существующей линейки программного обеспечения. Рассмотрены некоторые программные продукты и способы взаимодействия их с информационными проектными технологиями.
\end{abstract}

Ключевые слова: проектные технологии, информационное моделирование, архитектура зданий

BIM (англ. Building Information Modeling), или технологии информационного моделирования зданий и сооружений, - это далеко не новое явление, о котором слышала, наверное, на нынешний момент каждая уважающая себя организация хоть как-то связанная с проектированием и строительством зданий и сооружений. [1] Технологиям информационного моделирования находят с каждым годом все более широкое применение в разных странах. Но несмотря на это мало кто может грамотно перестроить все проектные процессы и произвести верное и полное внедрение ВIM в проектное производство своих фирм, изменив саму суть методологии ведения проектных процессов. (рисунок 1).

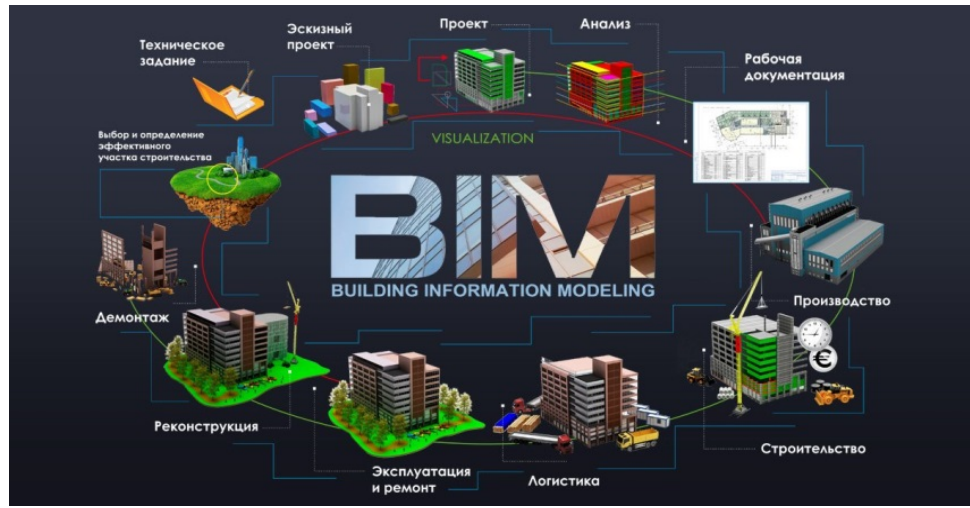

Рисунок 1. Схема внедрения ВIM.

Многие архитектурно-строительные компании все еще не уверены в преимуществе BIM технологий в основном из-за отсутствия опыта внедрения, грамотных специалистов, финансовых возможностей, либо из-за консервативной точки зрения на проектные и строительные процессы в целом, в котором ВIM зачастую игнорируется. В особенности на этапе 
концептуального или эскизного проектирования архитекторы игнорируют использование подходов BIM, полагаясь на стандартные методы моделирования используя такие программные обеспечения как 3DsMAX, SketchUp, AutoCAD.

Многие архитекторы интегрируют параметрическое моделирование и визуальное программирование в рабочие процессы своих проектов, используя такие приложения, как Grasshopper, Rino, Dynamo. Однако если бы они изначально применяли частично данную технологию, то многие рабочие моменты можно было бы решить уже на начальных этапах проектирования. (рисунок 2).

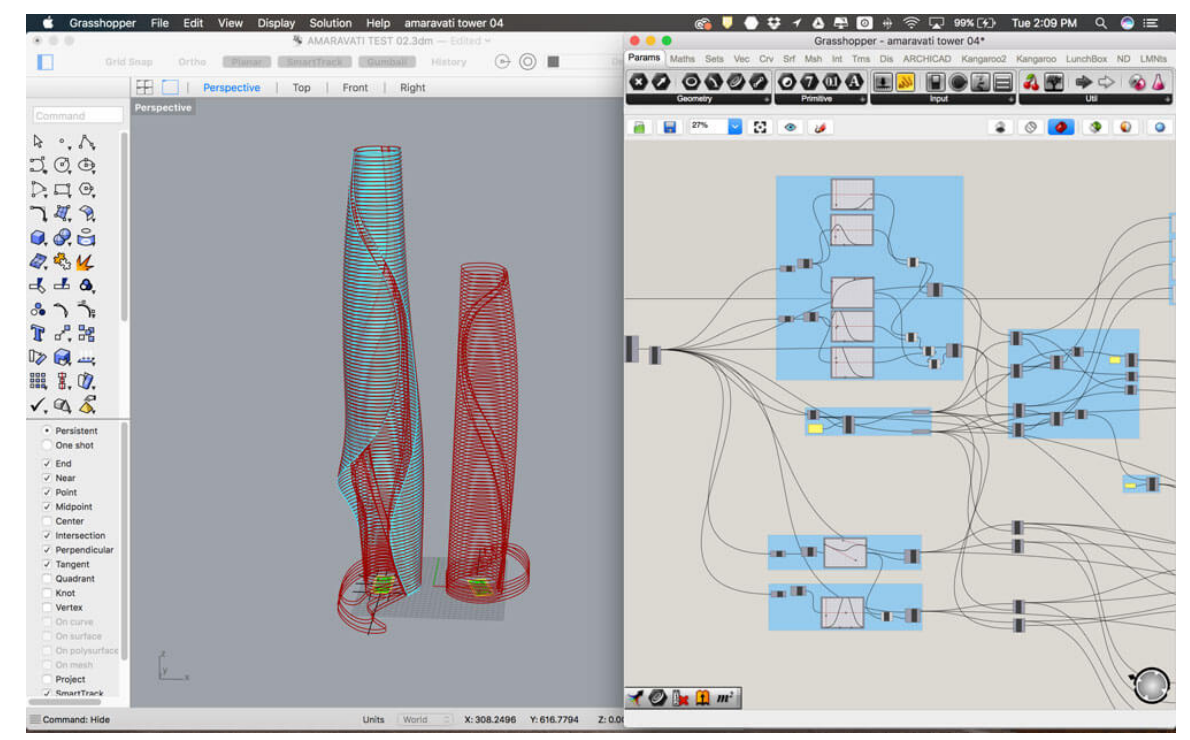

Рисунок 2 - Рабочий процесс в программах Grasshopper и Rino.

В основе начального процесса BIM проектирования, как правило, лежит параметрическое моделирование. Технически это значит, что BIM модель, ее типы и экземпляры имеют определённые параметры и настройки: ширина, длина, высота, тип изделия, массу, площадь, стоимость и т.д. [2]. То есть каждый объект в 3D модели имеет настройки имитирующие реальные свойства и поведение строительного материала, путем установки значений свойств всех параметров. Тогда, как консервативный пользователь вручную моделирует всю геометрию, а все свойства и параметры и вовсе отображает лишь в визуальном плане, либо в сторонних приложениях, что приводит к постоянным недопониманиям между смежными разделами и ярко выраженным коллизиям. (рисунок 3)

Хотя ВIM сильно зависит от параметрической функциональности, он в основном используется на объектном уровне, где пользователь контролирует создание каждого объекта в отдельности собирая это все в общую логически составленную цепочку, поэтому BIM модель, больше похожа на сборку самостоятельных объектов, с полным контролем как над общей формой, так и над единым объектом модели.

Основными преимуществами информационного моделирования можно аргументировать тем, что 3D модель полностью структурирована, с четко заданной информацией. Через моделирование и параметризацию 3D объектов, 
создается информация о самом проекте, а также дополнительная информация свойств элементов и экземпляров, из которых состоит основная модель.

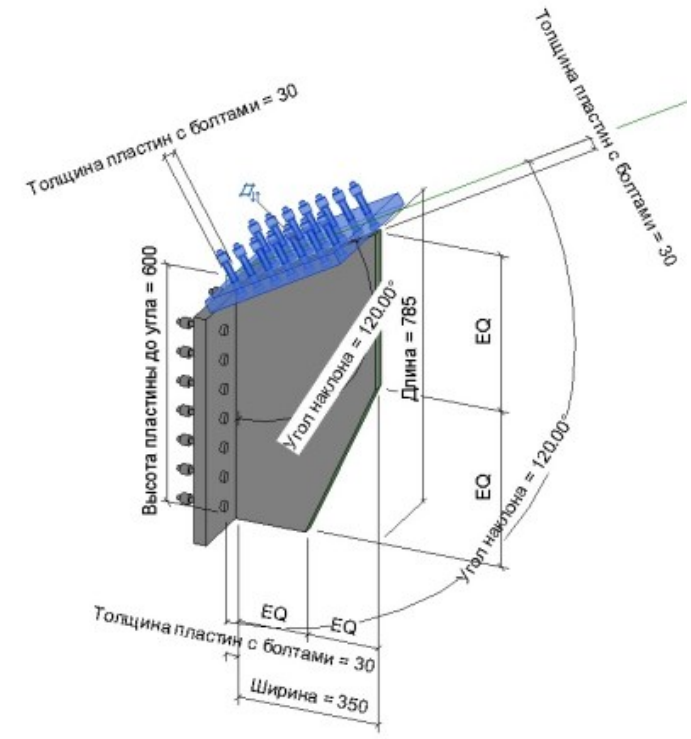

\section{Рисунок 3 - Параметрическое семейство Revit}

Кроме того, правильно структурированная ВIM модель способна выдать всю разрешительную документацию, в соответствии с нормативным оформлением того или иного государства. Прежде всего в виде 2D чертежей, такие как планы, фасады, разрезы, спецификации, узлы и т.д. которые попрежнему необходимы для получения разрешения на строительство и для реализации строительных работ.

Параметрический подход моделирования является более гибким, например Rhino, MicroStation и CATIA для Grasshopper. Когда геометрия полностью сгенерирована у пользователя есть возможность экспортировать свои параметрические модели в разные программные обеспечения на основе ВIM и продолжить работу уже там [3].

Например, стена в ВIM модели, является системным элементом, который получает полный состав свойств, включая утеплитель и отделку. (рисунок 4)

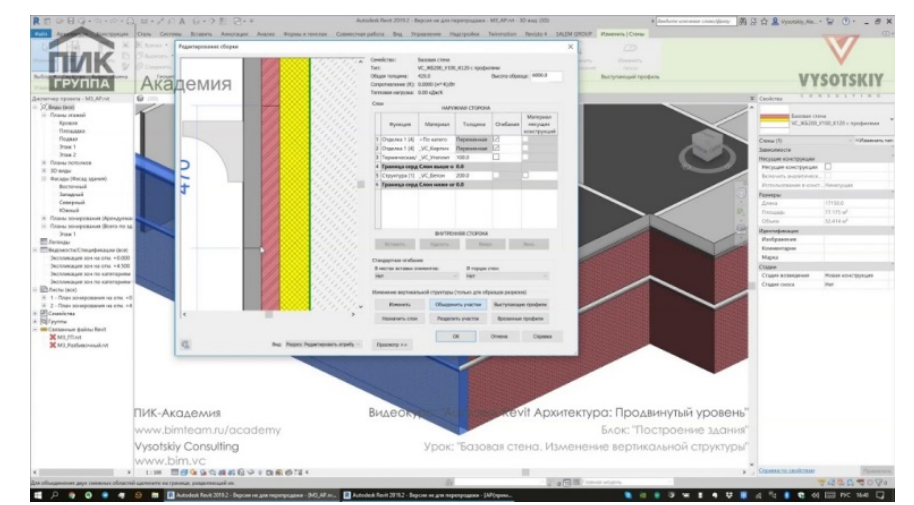

\section{Рисунок 4 - Параметры системного семейства стены в Revit}

BIM проектирование, ориентированно в основном, на геометрию модели. Хотя и сама модель привязана к внешним аналитическим инструментам, которые представляют собой различные спецификации или графики, для 
оценки различных критериев эффективности проекта, таких как расчет инсоляции, аэрации или потребление энергии, все же сам процесс по своей сути сосредоточен на основной 3D модели и на ее элементах.

Полученная геометрия содержит мало информации на начальном этапе проектирования и имеет не большие отличия от модели, созданной в традиционных системах САПР. Однако ВІМ подход так же удобен тем, что есть возможность работать в едином формате IFC (Industry Foundation Classes) формат данных с открытой спецификацией, которая не контролируется ни одной компанией или группой компаний. Данный Формат файла был создан компанией building SMART (International Alliance for Interoperability, IAI) для упрощения взаимодействия в строительной индустрии.

Недавно была запущена программа OpenBIM. (url: Buildingsmart.com/openbim), при поддержке BuildingSMART и некоторых софтверных компаний такие как Tekla, Graphisoft и Nemetschek.v OpenBIM ориентирована на упрощение обменом данных и информацией на основе формата файла IFC, независимо от используемого программного обеспечения $[4]$.

Уменьшение существующих разрывы между 3D моделью, созданной на основе параметрического моделирования и программного обеспечения для создания ВIM моделей, для проектировщиков становится серьезной задачей. Одним из подходов является - использование ВIM совместимых данных, а именно форматы для передачи более насыщенных моделей между обеими методологиями. Параметрическую модель можно использовать, для генерации входных файлов и данных, в формате IFC, которые в дальнейшем можно импортировать в программу на основе ВIM [5].

Обмен моделями через IFC по-прежнему будут развиваться, особенно когда станет доступно больше IFC-совместимых программных фреймворков с открытым исходным кодом.

Например, Система «ANAR +» с открытым исходным кодом (Labelle et al, 2009) генерирует скрипты из параметрической модели, обрабатывает данные (Reas \& Fry, 2007) и включает возможность определения собственных настроек и под настройками BIM модели. Работает это все, как система в виде GDLсовместимых скриптов, которые используются при определении параметрических объектов в ArchiCAD. То есть данный подход заключается не только в обмене 3D моделью или его элементами, а в передаче данных, системных настроек, переносе данных с диспетчера проекта (листы с чертежами, спецификации, аннотации, ведомости и т.д.).

В заключении можно сказать, что создание цифровой модели больше полагается на парадигмы параметрического проектирования, где замысел проекта и внешние информационные потоки могут быть заимствованы, для создания информационной модели. Программное обеспечение делает их совместимыми с системами BIM, преимущества обоих подходов могут дополнять друг друга и объединены в более цельный цифровой рабочий процесс, где нет определённой конкретной программы или инструмента, но информационные потоки находятся под контролем. 


\section{Заключение и выводы.}

В заключении можно сказать, что создание цифровой модели больше полагается на парадигмы параметрического проектирования, где замысел проекта и внешние информационные потоки могут быть заимствованы, для создания информационной модели. Программное обеспечение делает их совместимыми с системами BIM, преимущества обоих подходов могут дополнять друг друга и объединены в более цельный цифровой рабочий процесс, где нет определённой конкретной программы или инструмента, но информационные потоки находятся под контролем.

Литература:

1. Козлов И.М. Оценка экономической эффективности внедрения информационного моделирования зданий // АМІТ: электрон. журн. 2009. № 3(8)

2. Eastman, C., Teichholz, P., Sacks, R., \& Liston, K. BIM Handbook - A Guide to Building Information Modeling for Owners, Managers, Designers, Engineers, and Contractors // Справочник BIM, изд. John Wiley and Sons Нью-Джерси - 2011- C 648.

3. Дронов Д.С., Киметова Н.Р., Ткаченкова В.П. Проблемы внедрения BIМтехнологий в России // Международный научный журнал «Синергия наук», 2017, № 10 - c. 529-529.

4. A Russian example of the OPEN BIM approach. I. Viner-Usmanova. Case study. Rhythmic Gimnastics Center in the Luzhniki Complex. 2019. URL: https://graphisoft.com/pt/case-studies/the-center-for-rhythmic-gymnastics (дата обращения 10.04.2021)

5. Mirtschin, J. Engaging Generative BIM Workflows. Collaborative Design of Lightweight Structures - LSAA. // изд. Сидней. Австралия - 2011- С 8.

Abstract. The paper discusses the complexities of the transition to parametric design of architectural companies associated with the features of the existing line of software. Some software products and ways of their interaction with information design technologies are considered.

Key words: design technology, information modeling, building architecture

Статья отправлена: 22.04.2021 г.

(C) Шадыбеков T.X. 